\title{
Clinical outcome of Descemet stripping automated endothelial keratoplasty in 18 cases with iridocorneal endothelial syndrome
}

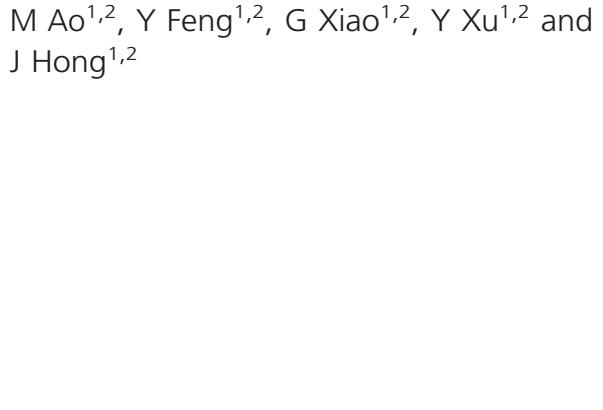

relatively poor. Immediate postoperative complications, postkeratoplasty glaucoma surgery, thicker corneal parameters, and a shallow anterior chamber were all associated with graft failure.

Eye (2018) 32, 679-686; doi:10.1038/eye.2017.282; published online 15 December 2017

\section{Introduction}

Iridocorneal endothelial (ICE) syndrome is a disease of unknown aetiology. ${ }^{1}$ Abnormal endothelial cell function and a reduced endothelial cell number ultimately result in corneal endothelial failure and irreversible corneal edema. ${ }^{2,3}$ Keratoplasty is indicated for the treatment of irreversible corneal edema in ICE syndrome. Several case series have been reported for penetrating keratoplasty (PK) in eyes with ICE syndrome, ${ }^{4-9}$ including a total of 63 cases, which had an average mean follow-up of 4.6 years. According to these reports, the average graft failure rate was $33 \%,{ }^{4-9}$ and the average rejection rate was $30 \%{ }^{7-9}$

Endothelial keratoplasty (EK) is a development in the field of corneal transplantation that has profound significance because of its minimally invasive design. ${ }^{10-14}$ There have been a few case series on the clinical outcomes of EK in patients with ICE syndrome. ${ }^{9,15-18}$ Because of the relative rarity of ICE syndrome, the sample sizes in these studies were relatively small (ranging from 3 to 12 grafts), which was insufficient for intensive discussions on the longitudinal endothelial cell counts, estimated graft survival or risk factors for graft failure.

We conducted this retrospective study and reviewed the records of 18 consecutive Chinese patients with ICE syndrome. Postoperative
${ }^{1}$ Department of Ophthalmology, Peking University Third Hospital, Beijing, China

${ }^{2}$ Beijing Key Laboratory of Restoration of Damaged Ocular Nerve, Beijing, China

Correspondence:

J Hong, Department of Ophthalmology, Peking University Third Hospital, Number Forty-Nine North Garden Road Haidian District, Beijing, 86100191 , China

Tel: +86-010-82266500; Fax: +86-10-82089951. E-mail: hongjing1964@sina. com

Received: 23 May 2017 Accepted in revised form: 25 October 2017 Published online: 15 December 2017 
complications, graft survival, longitudinal endothelial cell counts, corneal thickness, anterior chamber depth (ACD), and potential risk factors for graft failure after Descemet stripping automated endothelial keratoplasty (DSAEK) were analysed.

\section{Subjects and methods}

A retrospective review was performed in the corneal transplant database containing all operative and followup data of patients with DSAEK, who were managed at our hospital from January 2008 to December 2015.

Consecutive eyes with ICE syndrome and a minimum of 6 months of follow-up were selected for analysis. Case records were reviewed for the following information: demographics (age at the time of keratoplasty, sex); history of ocular surgery, duration of follow-up; prekeratoplasty best-corrected visual acuity (BCVA) and postkeratoplasty BCVA at 1, 3, 6, and 12 months; glaucoma treatment; additional intraoperative procedures at the time of keratoplasty; postkeratoplasty intraocular pressure (IOP) at 1, 3, 6, and 12 months; postkeratoplasty glaucoma treatment; postoperative complications; and graft survival.

The patients provided informed consent for the use of their medical records in the research. The study was approved by the Peking University Third Hospital Institutional Review Board and adhered to the tenets of the Declaration of Helsinki.

The diagnosis of ICE was based on the presence of at least two of the following three principal diagnostic criteria: ${ }^{1}$ unilateral corneal edema caused by an abnormality of the corneal endothelium, or typical unilateral hammered-silver appearance of the posterior cornea; iris atrophy and peripheral anterior synechiae (PAS); or other iridocorneal adhesions.

\section{Surgical procedures}

All DSAEK operations were performed by a single surgeon $(\mathrm{JH})$. The surgery was performed following the standard procedures for DSAEK combined with the suture pull-through insertion technique. ${ }^{19}$ Donor tissue was prepared by a Moria automated lamellar therapeutic keratoplasty microkeratome equipped with a $400 \mu \mathrm{m}$ head and associated artificial AC (Moria Inc. Doylestown, PA, USA). The graft diameter was dependent on each patient's specific requirements and ranged from 7.5 to $8.0 \mathrm{~mm}$. Additional intraoperative procedures at the time of keratoplasty included lens extraction by phacoemulsification (if necessary) and synechiolysis (in all cases).

\section{Postoperative management}

Postoperatively, patients were routinely evaluated at 1 day, 1 week, 1 month, 3 months, 6 months, and 12 months. Topical corticosteroid (prednisolone acetate, $1.0 \%$, Allergan Inc., Irvine, CA, USA), levofloxacin eye drops (0.5\%, Santen Co. Ishikawa, Japan), and cyclosporin eye drops (1\%, North China Pharmaceutical Company, Ltd., Shijiazhuang, Hebei Province, China) were administered four times daily for 1 month. The dosage was reduced gradually as clinically indicated. A maintenance regimen of prednisolone acetate once daily was used after 12 months.

\section{Main outcome measures}

Visual outcomes BCVA was evaluated by the Snellen chart and then converted into LogMAR values for statistical analysis. If a patient was unable to read the standard chart, even at extremely close distances, finger counting and hand motion were tested.

\section{Slit-lamp biomicroscope examination A slit-lamp} biomicroscope (Haag-Streit 990, Courty Haag-Streit AG, Bern, Switzerland) was used to evaluate the anterior segment and graft survival. High-plus handheld condensing lenses (+90 D or $+78 \mathrm{D})$ were used to observe the posterior segment postoperatively. Graft rejection was defined as described in detail previously. ${ }^{20}$ Primary graft failure was defined as an edematous donor lenticule that never improved after surgery. Secondary graft failure (SGF) was defined as irreversible progressive corneal edema after an initial period of corneal clarity after transplantation.

Parameters of the anterior segment Corneal thickness and ACD were determined by the anterior segment optical coherence tomography (AS-OCT) system (VISANTE OCT MODEL 1000, ANTERIOR SEGMENT IMAGING; Carl Zeiss Meditec, Inc., Dublin, CA, USA). Central corneal thickness (CCT), graft thickness (GT) and anterior recipient corneal bed thickness (ARCBT) were measured using LASIK flap tool software. ${ }^{21}$ Pachymetry values were excluded from analysis in the presence of irreversible edema and corneal decompensation.

Endothelial cell density Preoperative donor ECD was obtained from eye bank donor documentation as counted with a specular microscope (HAI EB-3000 XYZ; HAI Laboratories, Lexington, MA, USA). Postoperative ECD was measured with a ConfoScan 4 microscope (Heidelberg retinal tomography, HRT3, Heidelberg Engineering, Heidelberg, Germany). Following the manufacturer's recommended procedure, a single trained 

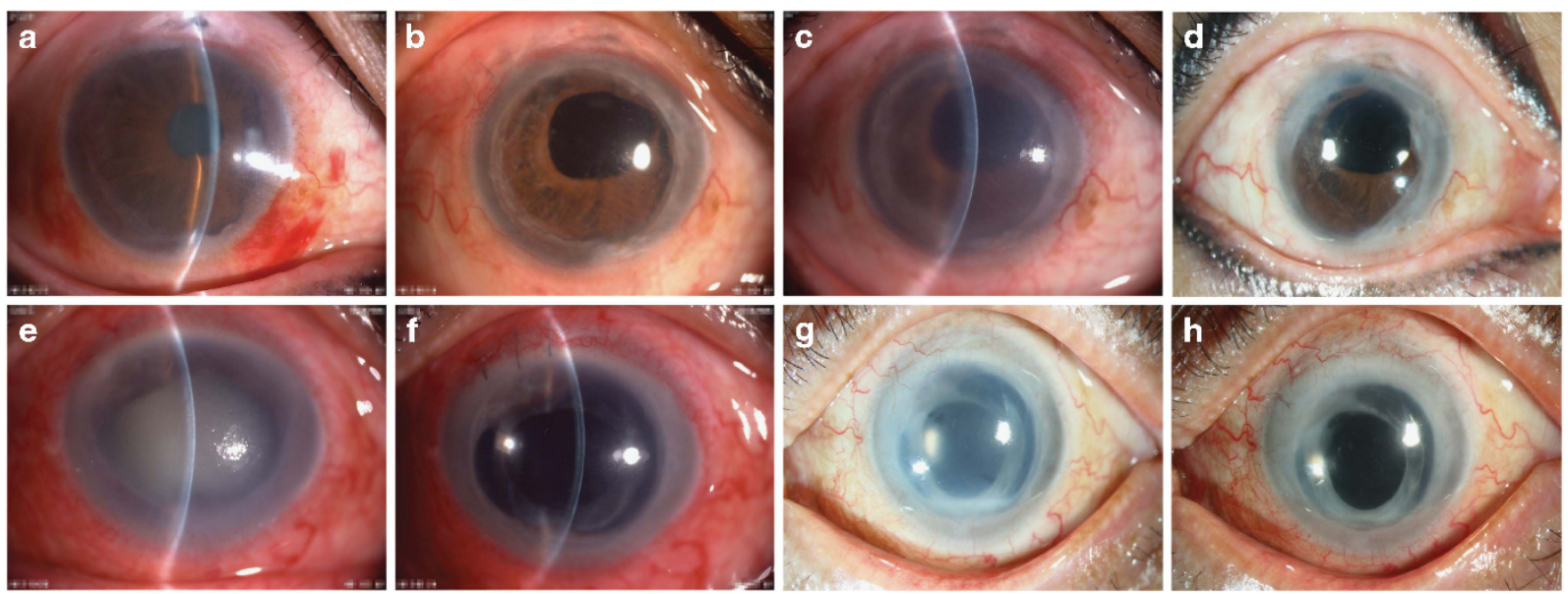

Figure 1 Slit lamp photographs of the two patients requiring repeat DSAEK. (a) A 48-year-old female patient with a history of trabeculectomy suffered from irreversible corneal edema for ICE syndrome. (b) She received successful DSAEK surgery combined with cataract surgery. (c) The female patient was diagnosed with secondary graft failure (SGF) at 9 months after the first DSAEK procedure. (d) The patient underwent a second DSAEK procedure and corneal clarity was restored postoperatively. (e) A 71-year-old male patient was diagnosed as ICE syndrome. (f) He underwent successful DSAEK surgery combined with cataract surgery. (g) The male patient was diagnosed with SGF at 15 months after the first DSAEK procedure. (h) The patient underwent a successful second DSAEK surgery.

technician (AHD) manually identified the cell centres and the exact perimeter of the cells being counted on the confocal images. Endothelial cell loss (ECL) was calculated by subtracting the central ECD determined at every review after surgery from the preoperative central ECD measured by the eye bank. Then, the ECL number was divided by the preoperative ECD and multiplied by 100 to give the percentage of ECL (ECL \%). Irreversible corneal edema was considered the endpoint for ECD measurement and the value was excluded from the following analysis.

\section{Statistical analysis}

The statistical analysis was performed using the SPSS software version 16.0 (SPSS, Inc., Chicago, IL, USA). The distribution of the data was assessed for normality using the Kolmogorov-Smirnoff-Lillefors test. Descriptive statistics for normally distributed continuous variables were reported as the mean $\pm S D$. Frequency distributions and percentages were used for categorical variables.

Continuous variables were compared using a two-tailed two-sample $t$-test or a two-tailed paired $t$-test when the data exhibited a normal distribution. The Wilcoxon ranksum test was used for data with non-normal distributions. A one-way ANOVA was used to analyse the differences within longitudinal data that exhibited a normal distribution with homogeneity of variance. Categorical variables were compared using $\chi^{2}$-analysis. For small sample sizes $(n<5)$, the Fisher's exact test was used. For outcomes in which 1 group had 0 events, no comparative statistics were reported. A Kaplan-Meier survival analysis was performed to evaluate the SGF rate at various times. A Log-rank test in the Kaplan-Meier analysis and Cox proportional hazard regression were used to analyse the influences of potential risk factors on the incidence of graft failure. A significance level of $P=0.05$ was assumed throughout.

\section{Results}

Eighteen eyes of 18 patients met the inclusion criteria. The patients were all Chinese and consisted of 5 males and 13 females, with a mean age of $51.1 \pm 13.0$ years (age range, 27-72 years). Two eyes of two patients (Figure 1a-d) required 1 repeat DSAEK graft, for a total of 20 DSAEK operations on the 18 eyes. The mean follow-up per graft was $19.0 \pm 8.6$ months (range, 6-36 months).

Supplementary Table S1 summarizes the details of each patient.

\section{Immediate postoperative complications}

On the first night postoperatively, a markedly elevated IOP of 40-60 mm Hg (Goldmann applanation tonometer) was identified in 9 of the 20 grafts and part of the air bubble was released from the auxiliary incision. The IOP of these eyes had returned to the normal level by the 1-day review. Graft detachment (as determined by the presence of interface fluid between the lenticule and the anterior recipient bed) occurred in three grafts at the 1-day examination and required immediate intervention for rebubbling. All grafts reattached successfully. 


\section{Rejection}

There were no cases of documented rejection in our study.

\section{Postoperative glaucoma}

Four eyes required additional glaucoma treatment postkeratoplasty. Two eyes received topical medication alone, including one that had undergone trabeculectomy before DSAEK surgery. One eye with no history of glaucoma prior to keratoplasty required trabeculectomy with mitomycin C. One eye with a history of trabeculectomy underwent glaucoma drainage device surgery.

\section{Visual outcomes}

Before DSAEK surgery, BCVA was $1.51 \pm 0.55 \log$ MAR (Snellen equivalent, 20/667). Postoperatively, BCVA improved significantly and reached a maximum $0.57 \pm 0.34 \log$ MAR (Snellen equivalent, 20/80) (paired t-test, $P<0.0001$ ) at $5.5 \pm 2.1$ months (range, $3-12$ months). Visual acuity gains were not sustained, as reflected by signs of graft decompensation.

\section{Corneal edema and ACD measurement}

The functional endothelial grafts relieved corneal edema gradually. Statistically significant differences were detected in the comparison of longitudinal ARCBT values (one-way ANOVA, $P=0.043$ ). A post hoc multiple comparison indicated significant differences in ARCBT values between 1 week and 1 month $(P=0.012)$ and between 1 week and 3 months $(P=0.006)$, suggesting the relief of corneal edema. At 24 months, a statistically thicker ARCBT was detected compared with that at 1 month $(P=0.036)$ and 3 months $(P=0.024)$, indicating corneal edema recurrence despite graft survival. No significant changes were observed in terms of longitudinal CCT, GT and ACD (one-way ANOVA, $P=0.066,0.286$ and 0.996 ) (Table 1$)$.

\section{ECD and ECL\% over time}

The ECD values of 20 (100\%), 19 (95\%), 17 (89.4\%), 13 $(76.5 \%), 8(80 \%)$, and $4(57.1 \%)$ surviving grafts were measured at $1,3,6,12,18$, and 24 months, respectively. Endothelial cells of some survival grafts $(1,2,3,1$, and 2 at $3,6,12,18$, and 24 months, respectively) could not be identified in the photographs from confocal microscopy. Compared with the ECD of donor corneal tissues $\left(3342.2 \pm 287.0\right.$ cells $\left./ \mathrm{mm}^{2}\right)$, ECL during DSAEK surgery was statistically significant (paired $t$-test, $P<0.0001$ ), with an ECD value of $1897.6 \pm 745.4$ cells $/ \mathrm{mm}^{2}$ and an ECL\% of $43.7 \pm 20.5$ at 1 month. In the follow-up, ECD declined gradually (one-way ANOVA, $P=0.161$ ), with a value of $1793.6 \pm 755.7$ cells $/ \mathrm{mm}^{2}(\mathrm{ECL} \%$ of $47.1 \pm 21.1)$ at

3 months, $1618.1 \pm 604.3$ cells $/ \mathrm{mm}^{2}(\mathrm{ECL} \%$ of $52.3 \pm 15.9)$ at 6 months, $1421.9 \pm 650.8$ cells $/ \mathrm{mm}^{2}(\mathrm{ECL} \%$ of $58.1 \pm 16.6)$ at 12 months, $1265.1 \pm 844.1$ cells $/ \mathrm{mm}^{2}(\mathrm{ECL} \%$ of $63.1 \pm 22.6)$ at 18 months and $1148.2 \pm 1217.8$ cells $/ \mathrm{mm}^{2}$ (ECL\% of $69.3 \pm 29.7$ ) at 24 months (Table 1 and Figure 2).

\section{SGF and risk factors}

At a mean time of $17.6 \pm 8.6$ months postoperatively (range, 6-30 months), irreversible corneal edema occurred in 11 of the 20 grafts (55\%). These 11 grafts were diagnosed as SGF due to late endothelial cell failure. The mean estimated graft survival by Kaplan-Meier analysis (Figure 3) was 23.4 months (95\% confidence interval, 18.6-28.2 months). The probabilities of survival were $95.0 \%, 85.0 \%, 60.7 \%, 53.1 \%$, and $21.2 \%$ at $6,12,18,24$, and 30 months, respectively.

The follow-up duration was comparable between grafts with SGF and surviving grafts (two-sample $t$-test, $P=0.403$ ). Immediate postoperative complications (including air-bubble ventilation for elevated IOP and rebubbling for graft detachment) were more common in eyes exhibiting graft failure $\left(\chi^{2}\right.$-analysis, $\left.P=0.040\right)$. According to the univariate analysis, postkeratoplasty glaucoma surgery was suggested as a potential risk factor for SGF (Log-rank test, $P=0.023$ ) (Table 2). According to the Cox proportional hazard regression, postkeratoplasty glaucoma surgery emerged as an independent risk factor for SGF (hazard ratio 5.174, 95\% confidence interval 1.00-26.90, $P=0.051$ ) with a statistically significant regression equation (Forward stepwise, likelihood ratio, $P=0.029$ ).

\section{Anatomic indicators of SGF}

The ECD, ECL\% and parameters measured by AS-OCT in the 12-month follow-up were analysed for potential indicators of SGF (Supplementary Table S2). Compared with eyes without graft failure, those with graft failure showed a statistically thicker CCT at 1, 3, 6, and 12 months, (two-sample $t$-test, $P=0.027,0.012,0.009$, and 0.038 , respectively) and GT at 3, 6, and 12 months (twosample $t$-test, $P=0.042,0.007$, and 0.042 , respectively). The ACD values at 6 and 12 months were significantly lower in eyes exhibiting graft failure than they were in eyes with surviving grafts (two-sample $t$-test, $P=0.021$ and $P=0.031)$.

\section{Discussion}

In the current study, we report DSAEK in 18 consecutive Chinese patients with ICE syndrome, representing the largest sample size among similar studies. Our results 
Table 1 Anatomic parameters after Descemet stripping automated endothelial keratoplasty

\begin{tabular}{lccccccc}
\hline & \multicolumn{5}{c}{ Follow-up visit } \\
\cline { 2 - 7 } & 1 Week & 1 Month & 3 Months & 6 Months & 12 Months & 18 Months & 24 Months \\
\hline CCT $(\mu \mathrm{m})$ & $815.4 \pm 130.6$ & $711.8 \pm 103.8$ & $693.9 \pm 107.5$ & $738.8 \pm 181.4$ & $720.1 \pm 166.4$ & $677.8 \pm 105.4$ & $831.2 \pm 209.2$ \\
GT $(\mu \mathrm{m})$ & $225.6 \pm 83.7$ & $187.9 \pm 81.9$ & $176.8 \pm 81.2$ & $196.0 \pm 109.6$ & $162.9 \pm 80.9$ & $151.4 \pm 51.2$ & $228.5 \pm 147.6$ \\
ARCBT $^{\mathrm{a}}(\mu \mathrm{m})$ & $589.8 \pm 71.4$ & $523.9 \pm 42.7^{\mathrm{b}}$ & $517.7 \pm 47.7^{\mathrm{b}}$ & $542.8 \pm 88.9$ & $557.1 \pm 113.0$ & $526.3 \pm 84.9$ & $602.7 \pm 108.8$ \\
$\mathrm{ACD}^{(\mathrm{mm})}$ & $2.70 \pm 0.44$ & $2.70 \pm 0.47$ & $2.69 \pm 0.52$ & $2.67 \pm 0.56$ & $2.61 \pm 0.63$ & $2.77 \pm 0.49$ & $2.67 \pm 0.57$ \\
$\mathrm{ECD}^{\mathrm{c}}\left(\mathrm{cells} / \mathrm{mm}^{2}\right)$ & - & $1897.6 \pm 745.4$ & $1793.6 \pm 755.7$ & $1618.1 \pm 604.3$ & $1421.9 \pm 650.8$ & $1265.1 \pm 844.1$ & $1148.2 \pm 1217.8$ \\
$\mathrm{ECL}^{\S}(\%)$ & - & $43.7 \pm 20.5$ & $47.1 \pm 21.1$ & $52.3 \pm 15.9$ & $58.1 \pm 16.6$ & $63.1 \pm 22.6$ & $69.3 \pm 29.7$ \\
\hline
\end{tabular}

Abbreviations: ACD, anterior chamber depth; ARCBT, anterior recipient corneal bed thickness; CCT, central corneal thickness; ECD, endothelial cell density; ECL, endothelial cell loss; GT, graft thickness. The values are presented as the means \pm standard deviation (SD).

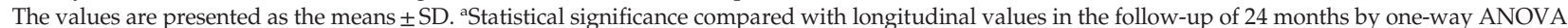
$(P=0.043)$. ${ }^{b}$ Satistical significance in the post hoc multiple comparison compared with the value at 1 week $(P=0.012)$ and 24 months $(P=0.006)$. ${ }^{c} C o n f o c a l$ scan microscopy data were available for 20 eyes at 1 month, 19 eyes at 3 months, 17 eyes at 6 months, 13 eyes at 12 months, 8 eyes at 18 months, and 4 eyes at 24 months.
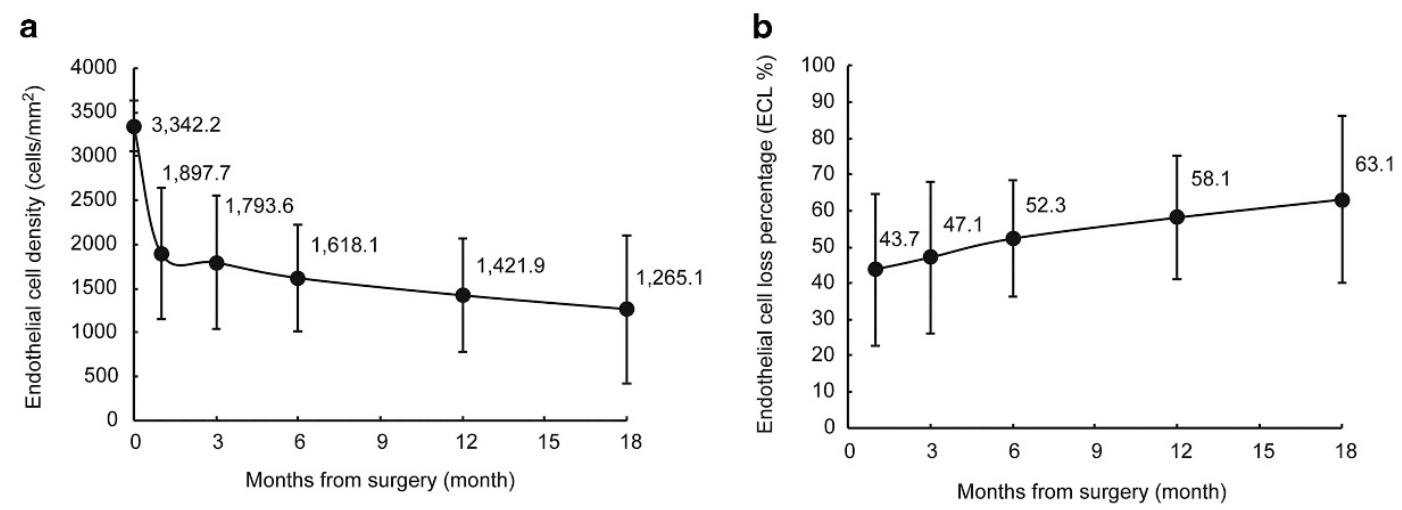

Figure 2 Decline of endothelial cell density (ECD) after DSAEK surgery. (a) Compared with the ECD of a donor cornea, which was designated ECD at 0 months, ECD decreased dramatically after DSAEK surgery. The most significant decline was detected at 1 month $(P<0.0001)$. There were $20,20,19,17$, and 10 surviving grafts at $1,3,6,12$, and 18 months, respectively. Although corneal edema was not detected, endothelial cells of 1, 2, 3, and 1 grafts could not be identified by confocal microscopy at 3, 6, 12, and 18 months. (b) Consistent with ECD decline, the endothelial cell loss percentage (ECL\%) was $43.7 \%$ at 1 month, and it increased gradually over the course of follow-up. The error bars depict the SD. mon, month after DSAEK surgery; $n$, number of observations at each follow-up visit.

showed a failure rate of $55 \%$, with a mean estimated survival of 23.4 months. Immediate postoperative complications, including air-bubble ventilation for markedly elevated IOP and rebubbling for graft detachment, were more common in eyes exhibiting graft failure. Postkeratoplasty glaucoma surgery was an independent risk factor associated with; SGF.

DSAEK in eyes with ICE syndrome is challenging. Severe iris atrophy, intra-operative IOP fluctuations, antedisplacement of the lens-iris diaphragm, difficult lenticule insertion and air tamponade were commonly encountered in our series. Cohesive viscoelastic Healon GV (Advanced Medical Optics, Santa Ana, CA, USA) with a better space-maintaining capacity was used to support the anterior chamber. Adequate synechiolysis was gently performed to create sufficient space for graft manipulation. The suture pull-through technique ${ }^{19}$ was used for donor insertion. When a stable air bubble could not be achieved, a very small amount of cohesive viscoelastic was injected under the centre of the graft. ${ }^{22}$ An air bubble was injected within the viscoelastic to form a bubble with a thin protecting shell.

After DSAEK surgery, the short-term clinical outcomes were acceptable in the eyes with ICE syndrome. Corneal edema was relieved in 1 to 3 months after DSAEK, with decreasing values of ARCBT. At 3 months, corneal edema had resolved to an optimum state. With restoration of corneal clarity, BCVA improved gradually and reached a maximum of $0.57 \log$ MAR (Snellen equivalent, 20/80) at 5 months, which is slightly poorer than that reported in previous studies (BCVA ranging from 20/50 to 20/20, as tested by the Snellen chart). ${ }^{15,17,18}$ The poor visual outcome in our series could be attributed to the relatively high rates of advanced glaucoma, which is an important visionlimiting comorbidity in patients with ICE syndrome. ${ }^{18}$

In our study, there was a significant decrease in ECD at 1 month, suggesting that the most significant ECL in DSAEK grafts occurred at the perioperative phase. Our 
data were similar to those reported by Fajgenbaum et al, ${ }^{17}$ with an ECL rate of $37 \%$ at 1 month. It has been agreed that the complicated anterior segment in eyes with ICE syndrome leads to great difficulty in the process of graft insertion and positioning, ${ }^{9,15-18}$ which is an important reason for the increased perioperative ECL rate. ${ }^{17}$ These

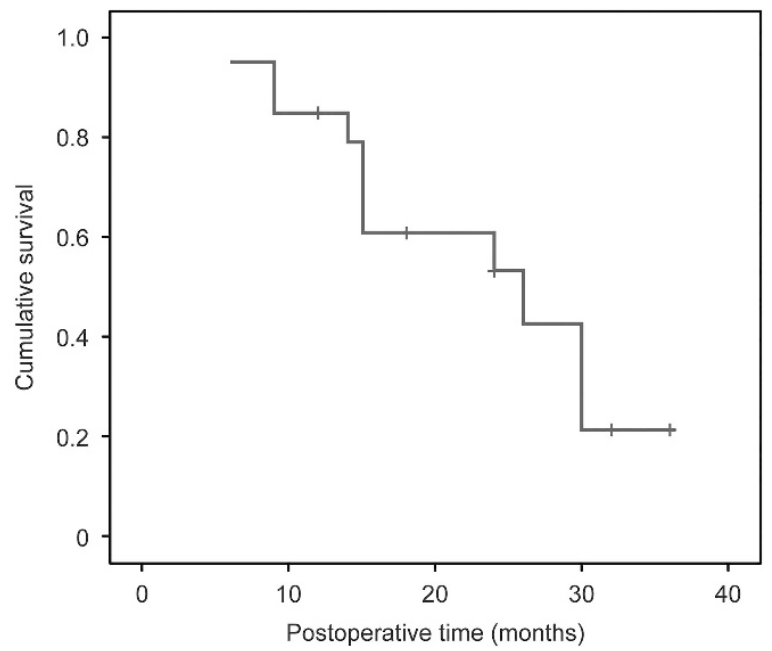

Figure 3 Kaplan-Meier survival curve demonstrating cumulative survival over time in months. All 20 grafts were assessed to determine the statistical likelihood of survival at various lengths of time. The mean estimated graft survival by Kaplan-Meier analysis was 23.4 months (95\% confidence interval, 18.628.2 months). The probability of survival was $95.0 \%$ at 6 months ( $n=20$ eyes with at least 6 months of follow-up), $85.0 \%$ at 12 months ( $n=17$ eyes with at least 12 months of follow-up), $60.7 \%$ at 18 months $(n=10$ eyes with at least 18 months of followup), $53.1 \%$ at 24 months ( $n=8$ eyes with at least 24 months of follow-up), and $21.2 \%$ at 30 months ( $n=4$ eyes with at least 30 months of follow-up). mon, month after DSAEK surgery. disadvantages are more pronounced in Chinese eyes that are characterized by a reduced ACD and high vitreous pressure. ${ }^{19,23}$

Long-term graft survival after DSAEK was poor in the eyes with ICE syndrome. In our study, 55\% (11 of 20) of the grafts exhibited SGF, with an estimated graft survival of 23.4 months. Fajgenbaum et al ${ }^{17}$ observed 7 of 9 grafts with SGF, with a mean graft survival of 19 months. A multicentre study including 12 eyes reported a graft failure rate of $33 \%$ (4 of 12 grafts) after DSAEK, with estimated mean survival rates of 4.5 and 4.7 years at two different sites. ${ }^{9}$ The variation in graft survival among these studies could be attributed to differences in the prevalence of prekeratoplasty glaucoma surgery (55\% in our study and $77 \%$ in the study conducted by Fajgenbaum et al $^{17}$ versus $34.5 \%$ in the multicentre study ${ }^{9}$ ), which is an independent risk factor for graft failure after DSAEK. ${ }^{24-26}$ Ethnicity could be another reason for the poor long-term outcome in our series. In the multicentre study with a relatively optimal survival rate, ${ }^{9}$ the percentage of Chinese patients was only $58.3 \%$. The biometrically shallow anterior chamber in Asian eyes ${ }^{23}$ results in greater surgical trauma to endothelial cells and the use of relatively little donor tissue, both of which were risk factors for graft failure. ${ }^{27,28}$

Immediate postoperative complications may accelerate the manifestation of SGF. Transient high IOP could affect the activity of endothelial cells, which may be exacerbated in the presence of other pathological factors in ICE syndrome and result in poor long-term survival. As the function of the IOP regulation system in eyes with ICE syndrome is too fragile to ventilate viscoelastic residue, postoperative IOP should be strictly monitored to manage

Table 2 Risk factors for secondary graft failure after DSAEK in eyes with ICE syndrome

\begin{tabular}{|c|c|c|c|c|}
\hline \multirow[t]{2}{*}{ Risk factor } & \multirow[t]{2}{*}{ Failed grafts $(\mathrm{N}=11)$} & \multirow[t]{2}{*}{ Surviving grafts $(\mathrm{N}=9)$} & \multicolumn{2}{|c|}{ P-values } \\
\hline & & & $\mathrm{P}_{1}^{\mathrm{a}}$ & $\mathrm{P}_{2}^{\mathrm{b}}$ \\
\hline Age at the time of keratoplasty (years) & $49.00 \pm 15.45$ & $55.56 \pm 9.52$ & 0.282 & - \\
\hline Duration of follow-up (months) & $18.55 \pm 9.45$ & $19.22 \pm 9.32$ & 0.874 & - \\
\hline Male/female & $2 / 9$ & $4 / 5$ & 0.202 & 0.712 \\
\hline Prekeratoplasty cataract surgery (\%) & $4(36.4)$ & $5(55.5)$ & 0.391 & 0.971 \\
\hline Prekeratoplasty glaucoma (\%) & $8(72.7)$ & $5(55.6)$ & 0.423 & 0.547 \\
\hline Topical medication $(\%)$ & $2(18.2)$ & $0(0.0)$ & 0.178 & 0.081 \\
\hline Underwent glaucoma surgery $(\%)$ & $6(54.5)$ & $5(55.5)$ & 0.964 & 0.781 \\
\hline Cataract surgery during keratoplasty (\%) & $3(27.3)$ & $2(22.2)$ & 0.795 & 0.888 \\
\hline Immediate postoperative complications & $9(81.8)$ & $3(33.3)$ & $0.040^{c}$ & 0.083 \\
\hline Markedly elevated IOP on the first night (\%) & $6(54.5)$ & $3(33.3)$ & 0.343 & 0.308 \\
\hline Received rebubbling $(\%)$ & $3(27.3)$ & $0(0.0)$ & - & 0.341 \\
\hline Postkeratoplasty glaucoma & $4(36.4)$ & $0(0.0)$ & - & 0.158 \\
\hline Topical medication (\%) & $2(18.2)$ & $0(0.0)$ & - & 0.815 \\
\hline Underwent glaucoma surgery (\%) & $2(18.2)$ & $0(0.0)$ & - & $0.023^{\mathrm{d}}$ \\
\hline
\end{tabular}

The data are presented as the proportions or the means $\pm \mathrm{SD}$ as appropriate for the variable. ${ }^{\mathrm{a}} \mathrm{For}$ comparisons between eyes with and without graft failure, $\chi^{2}$-analysis was used for categorical variables and a two-sample $t$-test was used for continuous variables. ${ }^{\mathrm{b}}$ Results of the Log-rank test in the Kaplan-Meier analysis. 'Statistical significance when comparing between eyes with and without graft failure. ${ }^{\mathrm{d}}$ Statistical significance for the Log-rank test in the KaplanMeier analysis. 
elevated pressure efficiently and reduce its impact. In addition, in our study, all grafts exhibited SGF after reattachment, which is consistent with previous studies on the complications of DSAEK. ${ }^{29,30}$ The endothelium may have been damaged while detached and the cell loss increased significantly in rebubbling, as shown by the attenuation or absence of endothelial cells according to histopathology. ${ }^{31}$ The surgical technique should be developed to avoid graft detachment in eyes with ICE syndrome.

Postkeratoplasty glaucoma surgery emerged as a risk factor for poor graft survival. A previous study on DSAEK for ICE syndrome indicated that adequate glaucoma control was the most important factor for achieving a good clinical outcome. ${ }^{18}$ In another study, postkeratoplasty glaucoma surgery was specifically assessed as a potential risk factor for graft failure after DSAEK or PK in eyes with ICE syndrome, ${ }^{9}$ but a positive result was not identified in the sample. It has been deduced that an increased influx of inflammatory mediators due to disruption of the blood-aqueous barrier or tube endothelial contact could cause severe damage to the endothelial cells of DSAEK grafts and lead to graft failure. ${ }^{24,25}$ In our series, irreversible corneal edema occurred in the two eyes that underwent glaucoma surgery immediately after the procedure, suggesting a direct adverse effect of postkeratoplasty glaucoma surgery on graft failure in eyes with ICE syndrome.

Postoperative CCT, GT, and ACD values may predict graft failure in eyes with ICE syndrome. Higher CCT and GT values within the first 6 months could be considered a sign of the prolonged relief of corneal edema and poor functional reserve of the endothelial graft, which are associated with poor graft survival. Moreover, the ACD value could be an indirect index of the severity of PAS. PAS recurrence was a manifestation of primary pathological progression postkeratoplasty ${ }^{16}$ and resulted in damage to nearby endothelial cells, both of which can cause a rapid loss of endothelial cells. Our results also indicated that a thinner donor graft, such as that in Descemet membrane endothelial keratoplasty (DMEK), may be advantageous, because its less contact with the peripheral iris. ${ }^{32}$ Factors such as the integrity of the lensiris diaphragm, intraoperative visualization, stability of the anterior chamber and difficulty in graft unfolding should be considered when planning DMEK for eyes with ICE syndrome. ${ }^{33-35}$ Ultra-thin DSAEK may be an alternative surgical method because of the ease of graft manipulation and attachment. ${ }^{36,37}$

In conclusion, the long-term outcome of DSAEK in eyes with ICE syndrome is relatively poor. Patients should be advised about the high probability of graft replacement at $\sim 2$ years postoperatively. Immediate postoperative complications, postkeratoplasty glaucoma surgery, thicker corneal parameters, and a shallow anterior chamber were associated with graft failure. Further clinical studies and histopathologic examinations are needed to determine the risk factors and their exact mechanisms for pronounced endothelial cell loss in eyes with ICE syndrome.

\section{Summary}

What was known before

- For the complicated comorbidity, keratoplasty in eyes with ICE syndrome is always a challenge to ophthalmologist.

- For the relative rarity of ICE syndrome, there are only a few case series studies on clinical outcomes of DSAEK in patients with ICE syndrome, and the sample size in these studies was relatively small.

What this study adds

- The long-term outcome of DSAEK in eyes with ICE syndrome is relatively poor.

- Patients should be advised a high probability of graft replacement at around 2 years postoperatively.

- Immediate postoperative complication, postkeratoplasty glaucoma surgery, thicker corneal parameters, and shallower anterior chamber were associated with graft failure.

\section{Conflict of interest}

The authors declare no conflict of interest.

\section{Acknowledgements}

This study was supported by the National Natural Science Foundation of China, No. 31271045.

\section{References}

1 Sacchetti M, Mantelli F, Marenco M, Macchi I, Ambrosio O, Rama P. Diagnosis and management of iridocorneal endothelial syndrome. BioMed Res Int 2015; 2015: 763093.

2 Hirst LW, Bancroft J, Yamauchi K, Green WR. Immunohistochemical pathology of the corneal endothelium in iridocorneal endothelial syndrome. Invest Ophthalmol Vis Sci 1995; 36: 820-827.

3 Malhotra C, Pandav SS, Gupta A, Jain AK. Phenotypic heterogeneity of corneal endothelium in iridocorneal endothelial syndrome by in vivo confocal microscopy. Cornea 2014; 33: 634-637.

4 Buxton JN, Lash RS. Results of penetrating keratoplasty in the iridocorneal endothelial syndrome. Am J Ophthalmol 1984; 98: 297-301.

5 Crawford GJ, Stulting RD, Cavanagh HD, Waring GO 3rd. Penetrating keratoplasty in the management of iridocorneal endothelial syndrome. Cornea 1989; 8: 34-40.

6 Chang PC, Soong HK, Couto MF, Meyer RF, Sugar A. Prognosis for penetrating keratoplasty in iridocorneal endothelial syndrome. Refract Corneal Surg 1993; 9: 129-132. 
7 DeBroff BM, Thoft RA. Surgical results of penetrating keratoplasty in essential iris atrophy. J Refract Corneal Surg 1994; 10: 428-432.

8 Alvim PT, Cohen EJ, Rapuano CJ, Chung CW, Pereira ML, Eagle RC Jr et al. Penetrating keratoplasty in iridocorneal endothelial syndrome. Cornea 2001; 20: 134-140.

9 Quek DT, Wong CW, Wong TT, Han SB, Htoon HM, Ho CL et al. Graft failure and intraocular pressure control after keratoplasty in iridocorneal endothelial syndrome. Am J Ophthalmol 2015; 160: 422-429.e1.

10 Anshu A, Price MO, Tan DT, Price FW Jr. Endothelial keratoplasty: a revolution in evolution. Surv Ophthalmol 2012; 57: 236-252.

11 Ple-Plakon PA, Shtein RM. Trends in corneal transplantation: indications and techniques. Curr Opin Ophthalmol 2014; 25: 300-305.

12 Park CY, Lee JK, Gore PK, Lim CY, Chuck RS. Keratoplasty in the United States: a 10-year review from 2005 through 2014. Ophthalmology 2015; 122: 2432-2442.

13 Wacker K, Baratz KH, Maguire LJ, McLaren JW, Patel SV. Descemet stripping endothelial keratoplasty for Fuchs' endothelial corneal dystrophy: five-year results of a prospective study. Ophthalmology 2016; 123: 154-160.

14 Price MO, Calhoun P, Kollman C, Price Jr FW, Lass JH. Descemet stripping endothelial keratoplasty: ten-year endothelial cell loss compared with penetrating keratoplasty. Ophthalmology 2016; 123: 1421-1427.

15 Price MO, Price FW Jr. Descemet stripping with endothelial keratoplasty for treatment of iridocorneal endothelial syndrome. Cornea 2007; 26: 493-497.

16 Huang T, Wang Y, Ji J, Gao N, Chen J. Deep lamellar endothelial keratoplasty for iridocorneal endothelial syndrome in phakic eyes. Arch Ophthalmol 2009; 127: 33-36.

17 Fajgenbaum MA, Hollick EJ. Descemet stripping endothelial keratoplasty in iridocorneal endothelial syndrome: postoperative complications and long-term outcomes. Cornea 2015; 34: 1252-1258.

18 Chaurasia S, Ramappa M, Garg P, Murthy SI, Senthil S, Sangwan VS. Endothelial keratoplasty in the management of irido-corneal endothelial syndrome. Eye 2013; 27: 564-566.

19 Hong Y, Peng RM, Wang M, Qu HQ, Hong J. Suture pullthrough insertion techniques for descemet stripping automated endothelial keratoplasty in Chinese phakic eyes: outcomes and complications(4). PLoS One 2013; 8: e61929.

20 Wu EI, Ritterband DC, Yu G, Shields RA, Seedor JA. Graft rejection following descemet stripping automated endothelial keratoplasty: features, risk factors, and outcomes. Am J Ophthalmol 2012; 153: 949-957.e1.

21 Di Pascuale MA, Prasher P, Schlecte C, Arey M, Bowman RW, Cavanagh HD et al. Corneal deturgescence after descemet stripping automated endothelial keratoplasty evaluated by Visante anterior segment optical coherence tomography. Am J Ophthalmol 2009; 148: 32-7 e1.

22 Peng RM, Hao YS, Chen HJ, Sun YX, Hong J. Endothelial keratoplasty: the use of viscoelastic as an aid in reattaching the dislocated graft in abnormally structured eyes. Ophthalmology 2009; 116: 1897-1900.

23 Yuen LH, He M, Aung T, Htoon HM, Tan DT, Mehta JS. Biometry of the cornea and anterior chamber in Chinese eyes: an anterior segment optical coherence tomography study. Invest Ophthalmol Vis Sci 2010; 51: 3433-3440.

24 Nahum Y, Mimouni M, Busin M. Risk factors predicting the need for graft exchange after descemet stripping automated endothelial keratoplasty. Cornea 2015; 34: 876-879.

25 Anshu A, Price MO, Price FW. Descemet's stripping endothelial keratoplasty: long-term graft survival and risk factors for failure in eyes with preexisting glaucoma. Ophthalmology 2012; 119: 1982-1987.

26 Decroos FC, Delmonte DW, Chow JH, Stinnett SS, Kim T, Carlson AN et al. Increased rates of descemet's stripping automated endothelial keratoplasty (DSAEK) graft failure and dislocation in glaucomatous eyes with aqueous shunts. $J$ Ophthal Vis Res 2012; 7: 203-213.

27 Ang M, Htoon HM, Cajucom-Uy HY, Tan D, Mehta JS. Donor and surgical risk factors for primary graft failure following descemet's stripping automated endothelial keratoplasty in Asian eyes. Clin Ophthalmol 2011; 5: 1503-1508.

28 Ishii N, Yamaguchi T, Yazu H, Satake Y, Yoshida A, Shimazaki J. Factors associated with graft survival and endothelial cell density after descemet's stripping automated endothelial keratoplasty. Sci Rep 2016; 6: 25276.

29 Suh LH, Yoo SH, Deobhakta A, Donaldson KE, Alfonso EC, Culbertson WW et al. Complications of descemet's stripping with automated endothelial keratoplasty: survey of 118 eyes at one institute. Ophthalmology 2008; 115: 1517-1524.

30 Price MO, Price FW Jr. Endothelial cell loss after descemet stripping with endothelial keratoplasty influencing factors and 2-year trend. Ophthalmology 2008; 115: 857-865.

31 Suh LH, Dawson DG, Mutapcic L, Rosenfeld SI, Culbertson WW, Yoo SH et al. Histopathologic examination of failed grafts in descemet's stripping with automated endothelial keratoplasty. Ophthalmology 2009; 116: 603-608.

32 Goldich Y, Artornsombidth P, Avni-Zauberman N, Perez M, Ulate R, Elbaz U et al. Fellow eye comparison of corneal thickness and curvature in descemet membrane endothelial keratoplasty and descemet stripping automated endothelial keratoplasty. Cornea 2014; 33: 547-550.

33 Price MO, Giebel AW, Fairchild KM, Price FW Jr. Descemet's membrane endothelial keratoplasty: prospective multicenter study of visual and refractive outcomes and endothelial survival. Ophthalmology 2009; 116: 2361-2368.

34 Terry MA. Endothelial keratoplasty: why aren't we all doing descemet membrane endothelial keratoplasty? Cornea 2012; 31: 469-471.

35 Hamzaoglu EC, Straiko MD, Mayko ZM, Sáles CS, Terry MA. The first 100 eyes of standardized descemet stripping automated endothelial keratoplasty versus standardized descemet membrane endothelial keratoplasty. Ophthalmology 2015; 122: 2193-2199.

36 Busin M, Madi S, Santorum P, Scorcia V, Beltz J. Ultrathin descemet's stripping automated endothelial keratoplasty with the microkeratome double-pass technique: two-year outcomes. Ophthalmology 2013; 120: 1186-1194.

37 Dickman MM, Kruit PJ, Remeijer L, van Rooij J, Van der Lelij A, Wijdh RH et al. A randomized multicenter clinical trial of ultrathin descemet stripping automated endothelial keratoplasty (DSAEK) versus DSAEK. Ophthalmology 2016; 123: 2276-2284. 